\title{
Criteria for Continuous-Variable Quantum Teleportation
}

\author{
Samuel L. Braunstein ${ }^{\dagger}$, Christopher A. Fuchs, and H. J. Kimble \\ Norman Bridge Laboratory of Physics, 12-33, California Institute of Technology \\ Pasadena, CA 91125, USA \\ ${ }^{\dagger}$ SEECS, University of Wales, Bangor LL57 1UT, UK \\ ${ }^{\dagger}$ Hewlett-Packard Labs, Math Group M48, Bristol BS34 8QZ, UK
}

10 April 1999

\begin{abstract}
We derive an experimentally testable criterion for the teleportation of quantum states of continuous variables. This criterion is especially relevant to the recent experiment of Furusawa et al. [Science 282, 706 (1998)] where an input-output fidelity of $0.58 \pm 0.02$ was achieved for optical coherent states. Our derivation demonstrates that fidelities greater than $1 / 2$ could not have been achieved through the use of a classical channel alone; quantum entanglement was a crucial ingredient in the experiment.
\end{abstract}

\section{Introduction}

What is quantum teleportation? The original protocol of Bennett et al. [1] specifies the idea with succinct clarity. The task set before Alice and Bob is to transfer the quantum state of a system in one player's hands onto a system in the other's. The agreed upon resources for carrying out this task are some previously shared quantum entanglement and a channel capable of broadcasting classical information. It is not allowed to physically carry the system from one player to the other, and indeed the two players need not even know each other's locations. One of the most important features of the protocol is that it must be able to work even when the state - though perfectly well known to its supplier, a third party Victor - is completely unknown to both Alice and Bob. Because the classical information broadcast over the classical channel can be minuscule in comparison to the infinite amount of information required to specify the unknown state, it is fair to say that the state's transport is a disembodied transport [2]. Teleportation has occurred when an unknown state $|\psi\rangle$ goes in and the same state $|\psi\rangle$ comes out.

But that is perfect teleportation. Recent experimental efforts [3, 4, 5, 6] show there is huge interest in demonstrating the phenomenon in the laboratory - a venue where perfection is unattainable as a matter of principle. The laboratory brings with it a new host of issues: if perfect teleportation is unattainable, when can one say that laboratory teleportation has been achieved? What appropriate criteria define the right to proclaim success in an experimental setting? Searching through the description above, there are several heuristic breaking points, each asking for quantitative treatment. The most important among these are:

1. The states should be unknown to Alice and Bob and supplied by an actual third party Victor.

2. Entanglement should be a verifiably used resource, with the possibility of physical transportation of the unknown states blocked at the outset. There should be a sense in which the output 
is "close" to the input - close enough that it could not have been made from information sent through a classical channel alone.

3. Each and every trial, as defined by Victor's supplying a state, should achieve an output sufficiently close to the input. When this situation pertains, the teleportation is called unconditional. (If that is impractical, conditional teleportation-where Alice and Bob are the arbiters of success - may still be of interest; but then, at the end of all conditioning, there must be a state at the output sufficiently close to the unknown input.)

To date only the Furusawa et al. experiment [3] has achieved unconditional experimental teleportation as defined by these three criteria. The Boschi et al. experiment [⿴囗十 fails to meet Criteria (1) and (2) because their Victor must hand off a (macroscopic) state-preparing device to Alice instead of an unknown state and because of a variety of low system efficiencies [3]. The Bouwmeester et al. experiment [5] fails to meet Criteria (2) and (3) because their output states-just before they are destroyed by an extra "verification" step - can be produced via communication through a classical channel alone [7]. In a similar vein, the Nielsen et al. experiment [6] fails to meet these criteria because there is no quantum entanglement shared between Alice and Bob at any stage of the process [8, 9].

But the story cannot stop there. Beside striving for simply better input-output fidelities or higher efficiencies, there are still further relevant experimental hurdles to be drawn from Ref. [1]:

4. The number of bits broadcast over the classical channel should be "minuscule" in comparison to the information required to specify the "unknown" states in the class from which the demonstration actually draws.

5. The teleportation quality should be good enough to transfer quantum entanglement itself instead of a small subset of "unknown" quantum states.

6. The sender and receiver should not have to know each other's locations to carry the process through to completion.

And there are likely still more criteria that would seem reasonable to one or another reader of the original protocol (depending perhaps upon the particular application called upon). The point is, these two lists together make it clear that the experimental demonstration of quantum teleportation cannot be a cut and dried affair. On the road toward ideal teleportation, there are significant milestones to be met and passed. Important steps have been taken, but the end of the road is still far from sight.

The work of the theorist in this effort is, among other things, to help turn the heuristic criteria above into pristine theoretical protocols within the context of actual experiments. To this end, we focus on Criterion 2 in the context of the Furusawa et al. experiment [3] where the quantum states of a set of continuous variables are teleported (as proposed in Refs. [10, 11]). The question is, by what means can one verify that Alice and Bob-assumed to be at fixed positions - actually use some quantum entanglement in their purported teleportation? How can it be known that they did not use the resource of a classical channel alone for the quantum state's transport? What milestone must be met in order to see this? Answering these questions fulfills a result already advertised in Ref. [3] and reported in the abstract of the present paper.

Our line of attack is to elaborate on an idea first suggested in Ref. [4]. A cheating Alice and Bob who attempt to make do with a classical channel alone, must gather information about the unknown quantum state if they are to have any hope of hiding their cheat. But then the limitations of quantum mechanics strike in a useful way. As long as the allowed set of inputs 
contains some nonorthogonal states, there is no measurement procedure that can reveal the state's identity with complete reliability. Any attempt to reconstruct the unknown quantum state will be flawed necessarily: information gathering about the identity of a state in a nonorthogonal set disturbs the state in the process [12, 13]. The issue is only to quantify how much disturbance must take place and to implement the actual comparison between input and output in an objective, operationally significant way. If the experimental match (or "fidelity") between the input and output exceeds the bound set by a classical channel, then some entanglement had to have been used in the teleportation process.

The remainder of the paper is structured as follows. In the following section, we discuss the motivation behind choosing the given measure of fidelity that we do. We stress in particular the need for a break with traditional quantum optical measures of signal transmission, such as signal-tonoise ratio, etc., used in the area of quantum nondemolition (QND) research [14, 15]. In Section 3, we derive the optimal fidelity that can be achieved by a cheating Alice and Bob whose teleportation measurements are based on optical heterodyning as in the experiment of Furusawa et al. [3]. This confirms that a fidelity of $1 / 2$ or greater is sufficient to assure the satisfaction of Criterion 2 in that experiment. We close in Section 4 with a few remarks about some open problems and future directions.

\section{$2 \quad$ Why Fidelity?}

Ideal teleportation occurs when an unknown state $|\psi\rangle$ goes into Alice's possession and the same state $|\psi\rangle$ emerges in Bob's. What can this really mean? A quantum state is not an objective state of affairs existing completely independently of what one knows. Instead it captures the best information available about how a quantum system will react in this or that experimental situation [16, 17]. This forces one to think carefully about what it is that is transported in the quantum teleportation process. The only option is that the teleported $|\psi\rangle$ must always ultimately refer to someone lurking in the background - a third party we label Victor, the keeper of knowledge about the system's preparation. The task of teleportation is to transfer what he can say about the system he placed in Alice's possession onto a system in Bob's possession: it is "information" in its purest form that is teleported, nothing more.

The resources specified for carrying out this task are the previously shared entanglement between Alice and Bob and a classical channel with which they communicate. Alice performs a measurement of a specified character and communicates her result to Bob. Bob then performs a unitary operation on his system based upon that information. When Alice and Bob declare that the process is complete, Victor should know with assurance that whatever his description of the original system was - his $|\psi\rangle$-it now holds for the system in Bob's possession. Knowing with assurance means that there really is a system that Victor will describe with $|\psi\rangle$, not that there was a system that he would have described with $|\psi\rangle$ just before Alice and Bob declared completion (i.e., as a retrodiction based upon their pronouncement) [7].

\footnotetext{
${ }^{1}$ On this bit of foundational theory, it seems most experimentalists can agree. See in particular page S291 of Zeilinger Ref. [16] where it is stated that: "The quantum state is exactly that representation of our knowledge of the complete situation which enables the maximal set of (probabilistic) predictions for any possible future observation. ... If we accept that the quantum state is no more than a representation of the information we have, then the spontaneous change of the state upon observation, the so-called collapse or reduction of the wave packet, is just a very natural consequence of the fact that, upon observation, our information changes and therefore we have to change our representation of the information, that is, the quantum state. From that position, the so-called measurement problem is not a problem but a consequence of the more fundamental role information plays in quantum physics as compared to classical physics."
} 
In any real-world implementation of teleportation, a state $\left|\psi_{\text {in }}\right\rangle$ enters Alice and Bob's dominion and a different state (possibly a mixed-state density operator) $\hat{\rho}_{\text {out }}$ comes out. As before, one must always keep in mind that these states refer to what Victor can say about the given system (see footnote 2$)$. The question that must be addressed is when $\left|\psi_{\text {in }}\right\rangle$ and $\hat{\rho}_{\text {out }}$ are similar enough to each other that Criterion 2 must have been fulfilled.

We choose to gauge the similarity between $\left|\psi_{\text {in }}\right\rangle$ and $\hat{\rho}_{\text {out }}$ by the "fidelity" between the two states. This is defined in the following wayt:

$$
F\left(\left|\psi_{\text {in }}\right\rangle, \hat{\rho}_{\text {out }}\right) \equiv\left\langle\psi_{\text {in }}\left|\hat{\rho}_{\text {out }}\right| \psi_{\text {in }}\right\rangle \text {. }
$$

This measure has the nice property that it equals 1 if and only if $\hat{\rho}_{\text {out }}=\left|\psi_{\text {in }}\right\rangle\left\langle\psi_{\text {in }}\right|$. Moreover it equals 0 if and only if the input and output states can be distinguished with certainty by some quantum measurement. The thing that is really important about this particular measure of similarity is hinted at by these last two properties. It captures in a simple and convenient package the extent to which all possible measurement statistics produceable by the output state match the corresponding statistics produceable by the input state.

To see what this means, take any observable (generally a positive operator-valued measure or POVM [17]) $\left\{\hat{E}_{\alpha}\right\}$ with measurement outcomes $\alpha$. If that observable were performed on the input system, it would give a probability density for the outcomes $\alpha$ given by

$$
P_{\text {in }}(\alpha)=\left\langle\psi_{\text {in }}\left|\hat{E}_{\alpha}\right| \psi_{\text {in }}\right\rangle \text {. }
$$

On the other hand, if the same observable were performed on the output system, it would give instead a probability density

$$
P_{\text {out }}(\alpha)=\operatorname{tr}\left(\hat{\rho}_{\text {out }} \hat{E}_{\alpha}\right) \text {. }
$$

A natural way to gauge the similarity of these two probability densities is by their overlap:

$$
\text { overlap }=\int \sqrt{P_{\text {in }}(\alpha) P_{\text {out }}(\alpha)} d \alpha \text {. }
$$

It turns out that regardless of which observable is being considered [18, 19],

$$
\text { overlap }^{2} \geq\left\langle\psi_{\text {in }}\left|\hat{\rho}_{\text {out }}\right| \psi_{\text {in }}\right\rangle \text {. }
$$

Moreover there exists an observable that gives precise equality in this expression 18, 19. In this sense, the fidelity captures an operationally defined fact about all possible measurements on the states in question.

Let us take a moment to stress the importance of a criterion such as this. It is not sufficient to attempt to quantify the similarity of the states with respect to a few observables. Quantum teleportation is a much more serious task than classical communication. Indeed it is a much more serious task than the simplest forms of quantum communication, as in quantum key distribution. In the former case, one is usually concerned with replicating the statistics of only one observable

\footnotetext{
${ }^{2}$ In order to form this quantity, we must of course assume a canonical mapping or identification between the input and output Hilbert spaces. Any unitary offset between input and output should be considered a systematic error, and ultimately taken into account by readjusting the canonical mapping. See Ref. 114, 15] for a misunderstanding of this point. The authors there state, "... fidelity does not necessarily recognize the similarity of states which differ only by reversible transformations. ... [This suggests] that additional measures are required ... based specifically on the similarity of measurement results obtained from the input and output of the teleporter, rather than the inferred similarity of the input and output states." As shown presently, the fidelity measure we propose does precisely that for all possible measurements, not just the few that have become the focus of present-day QND research.
} 
across a transmission line. In the latter case, one is concerned with reproducing the statistics of a small number of fixed noncommuting observables (the specific ones required of the protocol) for a small number of fixed quantum states (the specific ones required of the protocol). A full quantum state is so much more than the quantum measurements in these cases would reveal: it is a catalog for the outcome statistics of an infinite number of observables. Good quality teleportation must take that into account.

A concrete example can be drawn from the traditional concerns of quantum nondemolition measurement (QND) research. There a typical problem is how well a communication channel replicates the statistics of one of two quadratures of a given electromagnetic field mode [14, 15], and most often then only for assumed Gaussian statistics. Thinking that quantum teleportation is a simple generalization of the preservation of signal-to-noise ratio, burdened only in checking that both quadratures are transmitted faithfully, is to miss much of the point of teleportation. Specifying the statistics of two noncommuting observables only goes an infinitesimal way toward specifying the full quantum state when the Hilbert space is an infinite dimensional one [20, 21].

This situation is made acute by noticing that two state vectors can be almost completely orthogonal - and therefore almost as different as they can possibly be - while still giving rise to the same $x$ statistics and the same $p$ statistics. To see an easy example of this, consider the two state vectors $\left|\psi_{+}\right\rangle$and $\left|\psi_{-}\right\rangle$whose representations in $x$-space are

$$
\psi_{ \pm}(x)=\left(\frac{2 a}{\pi}\right)^{1 / 4} \exp \left((-a \pm i b) x^{2}\right)
$$

for $a, b \geq 0$. In $k$-space representation, these state vectors look like

$$
\tilde{\psi}_{ \pm}(k)=\left(\frac{a}{2 \pi}\right)^{1 / 4} \sqrt{\frac{a \pm i b}{a^{2}+b^{2}}} \exp \left(\frac{-a \mp i b}{4\left(a^{2}+b^{2}\right)} k^{2}\right) .
$$

Clearly neither $x$ measurements nor $p$ measurements can distinguish these two states. For, with respect to both representations, both wave functions differ only by a local phase function. However, if we look at the overlap between the two states we find:

$$
\left\langle\psi_{-} \mid \psi_{+}\right\rangle=\sqrt{\frac{a(a+i b)}{a^{2}+b^{2}}} .
$$

Taking $b \rightarrow \infty$, we can make these two states just as orthogonal as we please.

Suppose now that $\left|\psi_{+}\right\rangle$were Victor's input into the teleportation process, and-by whatever means - $\left|\psi_{-}\right\rangle$turned out to be the output. By a criterion that only gauged the faithfulness of the transmissions of $x$ and $p$ [14], this would be perfect teleportation. But it certainly isn't so!

Thus the justification of the fidelity measure in Eq. (1) as a measure of teleportation quality should be abundantly clear. But this is only the first step in finding a way to test Criterion 2. For this, we must invent a quantity that incorporates information about the teleportation quality of many possible quantum states. The reason for this is evident: in general it is possible to achieve a nonzero fidelity between input and output even when a cheating Alice and Bob use no entanglement whatsoever in their purported teleportation. This can come about whenever Alice and Bob can make use of some prior knowledge about Victor's actions.

As an example, consider the case where Alice and Bob are privy to the fact that Victor wishes only to teleport states drawn from a given orthogonal set. At any shot, they know they will be given one of these states, just not which one. Then, clearly, they need use no entanglement to "transmit" the quantum states from one position to the other. A cheating Alice need only perform 
a measurement $\mathcal{O}$ whose eigenstates coincide with the orthogonal set and send the outcome she obtains to Bob. Bob can use that information to resynthesize the appropriate state at his end. No entanglement has been used, and yet with respect to these states perfect teleportation has occurred.

This example helps define the issue much more sharply. The issue turns on having a general statement of what it means to say that Alice and Bob are given an unknown quantum state? In the most general setting it means that Alice and Bob know that Victor draws his states $\left|\psi_{\text {in }}\right\rangle$ from a fixed set $\mathcal{S}$; they just know not which one he will draw at any shot. This lack of knowledge is taken into account by a probability ascription $P\left(\left|\psi_{\text {in }}\right\rangle\right)$. That is:

All useful criteria for the achievement of teleportation must be anchored in whatever $\mathcal{S}$ and $P\left(\left|\psi_{\text {in }}\right\rangle\right)$ are given. A criterion is senseless if the states to which it is to be applied are not mentioned explicitly.

This makes it sensible to consider the average fidelity between input and output

$$
F_{\text {av }}=\int_{\mathcal{S}} P\left(\left|\psi_{\text {in }}\right\rangle\right) F\left(\left|\psi_{\text {in }}\right\rangle, \hat{\rho}_{\text {out }}\right) d\left|\psi_{\text {in }}\right\rangle,
$$

as a benchmark capable of eliciting the degree to which Criterion 2 is satisfied. If $\mathcal{S}$ consists of orthogonal states, then no criterion whatsoever (short of watching Alice and Bob's every move) will ever be able to draw a distinction between true teleportation and the sole use of the classical side channel. Things only become interesting when the set $\mathcal{S}$ consists of two or more nonorthogonal quantum states [4]: for only then will $F_{\text {av }}=1$ never be achievable by a cheating Alice and Bob.

By making the set $\mathcal{S}$ more and more complicated, we can define ever more stringent tests connected to Criterion 2. For instance, consider the simplest nontrivial case: take $\mathcal{S}=\mathcal{S}_{0}=$ $\left\{\left|\psi_{0}\right\rangle,\left|\psi_{1}\right\rangle\right\}$, a set of just two nonorthogonal states (with a real inner product $x=\cos \theta$ ). Suppose the two states occur with equal probability. Then it can be shown [12] that the best thing for a cheating Alice and Bob to do is this. Alice measures an operator whose orthogonal eigenvectors symmetrically bestride $\left|\psi_{0}\right\rangle$ and $\left|\psi_{1}\right\rangle$. Using that information, Bob synthesizes one of two states $\left|\tilde{\psi}_{0}\right\rangle$ and $\left|\tilde{\psi}_{1}\right\rangle$ each lying in the same plane as the original two states, but each tweaked slightly toward the other by an angle [22]

$$
\phi=\frac{1}{2} \arctan \left[\left(\frac{1+\sin \theta}{1-\sin \theta}+\cos 2 \theta\right)^{-1} \sin 2 \theta\right] .
$$

This (optimal) strategy gives a fidelity

$$
F_{\mathrm{av}}=\frac{1}{2}\left(1+\sqrt{1-x^{2}+x^{4}}\right) .
$$

Even in the worst case (when $x=1 / \sqrt{2}$ ), this fidelity is always relatively high - it is always above 0.933 [23].

This shows that choosing $\mathcal{S}_{0}$ to check for the fulfillment of Criterion 2 is a very weak test. For an example of the opposite extreme, consider the case where $\mathcal{S}$ consists of every normalized vector in a Hilbert space of dimension $d$ and assume that $\mathcal{S}$ is equipped with the uniform probability distribution (i.e., the unique distribution that is invariant with respect to all unitary operations). Then it turns out that the maximum value $F_{\text {av }}$ can take is [24]

$$
F_{\mathrm{av}}=\frac{2}{d+1} .
$$

For the case of a single qubit, i.e., $d=2$, Alice and Bob would only have to achieve a fidelity of $2 / 3$ before they could claim that they verifiably used some entanglement for their claimed teleportation. 
But, again, this is only if Victor can be sure that Alice and Bob know absolutely nothing about which state he inputs other than the dimension of the Hilbert space it lives in.

This last example finally prepares us to build a useful criterion for the verification of continuous quantum-variable teleportation in the experiment of Furusawa et al. [3]. For a completely unknown quantum state in that experiment would correspond to taking the limit $d \rightarrow \infty$ above. If Victor can be sure that Alice and Bob know nothing whatsoever about the quantum states he intends to teleport, then on average the best fidelity they can achieve in cheating is strictly zero! In this case, seeing any nonzero fidelity whatsoever in the laboratory would signify that unconditional quantum teleportation had been achieved.

But making such a drastic assumption for the confirmation set $\mathcal{S}$ would be going too far. This would be the case if for no other reason because any present-day Victor lacks the experimental ability to make good his threat. Any Alice and Bob that had wanted to cheat in the Furusawa et al. experiment would know that the Victor using their services is technically restricted by the fact that only a handful of manifestly quantum or nonclassical states have ever been generated in quantum optics laboratories [25]. By far the most realistic and readily available laboratory source available to Victor is one that creates optical coherent states of a single field mode for his test of teleportation. Therefore in all that follows we will explicitly make the assumption that $\mathcal{S}$ contains the coherent states $|\alpha\rangle$ with a Gaussian distribution centered over the vacuum state describing the probability density on that set. As we shall see presently, it turns out that in the limit that the variance of the Gaussian distribution approaches infinity-i.e., the distribution of states becomes ever more uniform - the upper bound for the average fidelity achievable by a cheating Alice and Bob using optical heterodyne measurements is

$$
F_{\text {av }}=\frac{1}{2} .
$$

Any average fidelity that exceeds this bound must have come about through the use of some entanglement.

\section{Optimal Heterodyne Cheating}

We now verify Eq. (13) within the context of the Furusawa et al. experiment. There, the object is to teleport an arbitrary coherent state of a finite bandwidth electromagnetic field. (The extension of the single mode theory of Ref. [1] to the multimode case is given in Ref. [26].) We focus for simplicity on the single mode case. The quantum resource used for the process is one that entangles the number states $|n\rangle$ of two modes of the field. Explicitly the entangled state is given by [27]

$$
|E\rangle_{\mathrm{AB}}=\frac{1}{\cosh r} \sum_{n=0}^{\infty}(\tanh r)^{n}|n\rangle_{\mathrm{A}}|n\rangle_{\mathrm{B}}
$$

where $r$ measures the amount of squeezing required to produce the entangled state.

In order to verify that entanglement was actually used in the experiment, as discussed in the previous section, we shall assume that the test set $\mathcal{S}$ is the full set of coherent states $|\beta\rangle$,

$$
|\beta\rangle=\exp \left(-|\beta|^{2} / 2\right) \sum_{0}^{\infty} \frac{\beta^{n}}{\sqrt{n !}}|n\rangle
$$

where the complex parameter $\beta$ is distributed according to a Gaussian distribution,

$$
p(\beta)=\frac{\lambda}{\pi} e^{-\lambda|\beta|^{2}} .
$$


Ultimately, of course, we would like to consider the case where Alice and Bob are completely ignorant of which coherent state is drawn. This is described by taking the limit $\lambda \rightarrow 0$ in what follows.

It is well known that the measurement optimal for estimating the unknown parameter $\beta$ when it is distributed according to a Gaussian distribution [28] is the POVM $\left\{\hat{E}_{\alpha}\right\}$ constructed from the coherent state projectors according to

$$
\hat{E}_{\alpha}=\frac{1}{\pi}|\alpha\rangle\langle\alpha|
$$

first suggested by Arthurs and Kelly [29]. This measurement is equivalent to optical heterodyning [30]. These points make this measurement immediately attractive for the present considerations. On the one hand, maximizing the average fidelity (as is being considered here) is almost identical in spirit to the state-estimation problem of Ref. [28]. On the other, in the Furusawa et al. experiment a cheating Alice who uses no entanglement actually performs precisely this measurement.

We therefore consider an Alice who performs the measurement $\left\{\hat{E}_{\alpha}\right\}$ and forwards on the outcome - i.e., the complex number $\alpha$ - to Bob. [ The only thing Bob can do with this information is generate a new quantum state according to some rule, $\alpha \rightarrow\left|f_{\alpha}\right\rangle$. Let us make no a priori restrictions on the states $\left|f_{\alpha}\right\rangle$. The task is first to find the maximum average fidelity $F_{\max }(\lambda)$ Bob can achieve for a given $\lambda$.

For a given strategy $\alpha \rightarrow\left|f_{\alpha}\right\rangle$, the achievable average fidelity is

$$
\begin{aligned}
F(\lambda) & =\int p(\beta)\left(\int p(\alpha \mid \beta)\left|\left\langle f_{\alpha} \mid \beta\right\rangle\right|^{2} d^{2} \alpha\right) d^{2} \beta \\
& =\int p(\beta)\left(\int \frac{1}{\pi}|\langle\alpha \mid \beta\rangle|^{2}\left|\left\langle f_{\alpha} \mid \beta\right\rangle\right|^{2} d^{2} \alpha\right) d^{2} \beta \\
& =\frac{\lambda}{\pi^{2}} \iint e^{-\lambda|\beta|^{2}} e^{-|\alpha-\beta|^{2}}\left|\left\langle f_{\alpha} \mid \beta\right\rangle\right|^{2} d^{2} \beta d^{2} \alpha \\
& =\frac{\lambda}{\pi^{2}} \int e^{-|\alpha|^{2}}\left\langle f_{\alpha}\left|\left(\int \exp \left(-(1+\lambda)|\beta|^{2}+2 \operatorname{Re} \alpha^{*} \beta\right)|\beta\rangle\langle\beta| d^{2} \beta\right)\right| f_{\alpha}\right\rangle d^{2} \alpha .
\end{aligned}
$$

Notice that the operator enclosed within the brackets in Eq. (21), i.e.,

$$
\hat{\mathcal{O}}_{\alpha}=\int \exp \left(-(1+\lambda)|\beta|^{2}+2 \operatorname{Re} \alpha^{*} \beta\right)|\beta\rangle\langle\beta| d^{2} \beta,
$$

is a positive semi-definite Hermitian operator that depends only on the real parameter $\lambda$ and the complex parameter $\alpha$. It follows that

$$
\left\langle f_{\alpha}\left|\hat{\mathcal{O}}_{\alpha}\right| f_{\alpha}\right\rangle \leq \mu_{1}\left(\hat{\mathcal{O}}_{\alpha}\right)
$$

where $\mu_{1}(\hat{X})$ denotes the largest eigenvalue of the operator $\hat{X}$.

With this, Bob's best strategy is apparent. For each $\alpha$, he simply adjusts the state $\left|f_{\alpha}\right\rangle$ to be the eigenvector of $\hat{\mathcal{O}}_{\alpha}$ with the largest eigenvalue. Then equality is achieved in Eq. (23), and it is just a question of being able to perform the integral in Eq. (21).

The first step in carrying this out is to find the eigenvector and eigenvalue achieving equality in Eq. (23). This is most easily evaluated by unitarily transforming $\hat{\mathcal{O}}_{\alpha}$ into something that is

\footnotetext{
${ }^{3}$ We caution however that the present considerations do not prove the optimality of heterodyne measurement for an arbitrarily adversarial Alice and Bob-they simply make it fairly plausible. Complete optimization requires the consideration of all POVMs that Alice can conceivably perform along with explicit consideration of the structure of the fidelity function considered here, not simply the variance of an estimator as in the state-estimation problem. More on this issue can be found in Ref. 32.
} 
diagonal in the number basis, picking off the largest eigenvalue, and transforming back to get the optimal $\left|f_{\alpha}\right\rangle$. (Recall that eigenvalues are invariant under unitary transformations.)

The upshot of this procedure is best illustrated by working backward toward the answer. Consider the positive operator

$$
\hat{P}=\int e^{-(1+\lambda)|\beta|^{2}}|\beta\rangle\langle\beta| d^{2} \beta .
$$

Expanding this operator in the number basis, we find

$$
\hat{P}=\pi \sum_{n=0}^{\infty}(2+\lambda)^{-(n+1)}|n\rangle\langle n| .
$$

So clearly,

$$
\mu_{1}(\hat{P})=\frac{\pi}{2+\lambda}
$$

Now consider the displaced operator

$$
\hat{Q}_{\alpha}=\hat{D}\left(\frac{\alpha}{1+\lambda}\right) \hat{P} \hat{D}^{\dagger}\left(\frac{\alpha}{1+\lambda}\right)
$$

where $\hat{D}(\nu)$ is the standard displacement operator [31]. Working this out in the coherent-state basis, one finds

$$
\begin{aligned}
\hat{Q}_{\alpha} & =\int e^{-(1+\lambda)|\beta|^{2}}\left|\beta+\frac{\alpha}{1+\lambda}\right\rangle\left\langle\beta+\frac{\alpha}{1+\lambda}\right| d^{2} \beta \\
& =\int \exp \left(-(1+\lambda)\left|\gamma-\frac{\alpha}{1+\lambda}\right|^{2}\right)|\gamma\rangle\langle\gamma| d^{2} \gamma \\
& =\exp \left(\frac{-|\alpha|^{2}}{1+\lambda}\right) \int \exp \left(-(1+\lambda)|\gamma|^{2}+2 \operatorname{Re} \alpha^{*} \gamma\right)|\gamma\rangle\langle\gamma| d^{2} \gamma \\
& =\exp \left(\frac{-|\alpha|^{2}}{1+\lambda}\right) \hat{\mathcal{O}}_{\alpha} .
\end{aligned}
$$

Using this in the expression for $F(\lambda)$ we find,

$$
\begin{aligned}
F(\lambda) & =\frac{\lambda}{\pi^{2}} \int \exp \left(-\left(1-\frac{1}{1+\lambda}\right)|\alpha|^{2}\right)\left\langle f_{\alpha}\left|\left(\hat{D}\left(\frac{\alpha}{1+\lambda}\right) \hat{P} \hat{D}^{\dagger}\left(\frac{\alpha}{1+\lambda}\right)\right)\right| f_{\alpha}\right\rangle d^{2} \alpha \\
& \leq \frac{1}{\pi} \frac{\lambda}{2+\lambda} \int \exp \left(-\frac{\lambda}{1+\lambda}|\alpha|^{2}\right) d^{2} \alpha \\
& =\frac{1+\lambda}{2+\lambda}
\end{aligned}
$$

Equality is achieved in this chain by taking

$$
\left|f_{\alpha}\right\rangle=D\left(\frac{\alpha}{1+\lambda}\right)|0\rangle=\left|\frac{\alpha}{1+\lambda}\right\rangle
$$

Therefore the maximum average fidelity is given by

$$
F_{\max }(\lambda)=\frac{1+\lambda}{2+\lambda}
$$


In the limit that $\lambda \rightarrow 0$, i.e., when Victor draws his states from a uniform distribution, we have

$$
F_{\max }(\lambda) \longrightarrow \frac{1}{2}
$$

as advertised in Ref. [3].

It should be noted that nothing in this argument depended upon the mean of the Gaussian distribution being $\beta=0$. Bob would need to minimally modify his strategy to take into account Gaussians with a non-vacuum state mean, but the optimal fidelity would remain the same.

\section{Conclusion}

Where do we stand? What remains? Clearly one would like to develop a toolbox of ever more stringent and significant tests of quantum teleportation - ones devoted not only to Criterion 2, but to all the others mentioned in the Introduction as well. Significant among these are delineations of the fidelities that must be achieved to ensure the honest teleportation of nonclassical states of light, such as squeezed states. Some work in this direction appears in Ref. [11, but one would like to find something more in line with the framework presented here. Luckily, a more general setting for this problem can be formulated [32] as it will ultimately be necessary to explore any number of natural verification sets $\mathcal{S}$ and their resilience with respect to arbitrarily adversarial Alice and Bob teams.

\section{Acknowledgments}

We thank Jason McKeever for suggesting the nice example in Eq. (6) and thank J. R. Buck and C. M. Caves for useful discussions. This work was supported by the QUIC Institute funded by DARPA via the ARO, by the ONR, and by the NSF. SLB was funded in part by EPSRC grant GR/L91344. CAF acknowledges support of the Lee A. DuBridge Fellowship.

\section{References}

[1] C. H. Bennett, G. Brassard, C. Crépeau, R. Jozsa, A. Peres, and W. K. Wootters, "Teleporting an Unknown Quantum State via Dual Classical and Einstein-Podolsky-Rosen Channels," Phys. Rev. Lett. 70, 1895 (1993).

[2] S. L. Braunstein, "A Fun Talk on Teleportation," available on the World Wide Web at http:// www. sees.bangor.ac.uk/ schmuel/tport.html.

[3] A. Furusawa, J. L. Sørensen, S. L. Braunstein, C. A. Fuchs, H. J. Kimble, and E. S. Polzik, "Unconditional Quantum Teleportation," Science 282, 706 (1998).

[4] D. Boschi, S. Branca, F. De Martini, L. Hardy, and S. Popescu, "Experimental Realization of Teleporting an Unknown Pure Quantum State via Dual Classical and Einstein-Podolsky-Rosen Channels," Phys. Rev. Lett. 80, 1121-1125 (1998).

[5] D. Bouwmeester, J.-W. Pan, K. Mattle, M. Eibl, H. Weinfurter, and A. Zeilinger, "Experimental Quantum Teleportation," Nature 390, 575-579 (1997).

[6] M. A. Nielsen, E. Knill, and R. Laflamme, "Complete Quantum Teleportation Using Nuclear Magnetic Resonance," Nature 396, 52-55 (1998). 
[7] S. L. Braunstein and H. J. Kimble, "A Posteriori Teleportation," Nature 394, 840-841 (1998).

[8] S. L. Braunstein, C. M. Caves, R. Jozsa, N. Linden, S. Popescu, and R. Schack, "Separability of Very Noisy Mixed States and Implications for NMR Quantum Computing," quant-ph/9811018.

[9] R. Schack and C. M. Caves, "Classical Model for Bulk-Ensemble NMR Quantum Computation," quant-ph/9903101.

[10] L. Vaidman, "Teleportation of Quantum States," Phys. Rev. A 49, 1473-1476 (1994).

[11] S. L. Braunstein and H. J. Kimble, "Teleportation of Continuous Quantum Variables," Phys. Rev. Lett. 80, 869-872 (1998).

[12] C. A. Fuchs and A. Peres, "Quantum State Disturbance vs. Information Gain: Uncertainty Relations for Quantum Information," Phys. Rev. A 53, 2038-2045 (1996).

[13] C. A. Fuchs, "Information Gain vs. State Disturbance in Quantum Theory," Fort. Phys. 46 535-565 (1998).

[14] T. C. Ralph, P. K. Lam, and R. E. S. Polkinghorne, "Characterizing Teleportation in Optics," quant-ph/9903003.

[15] T. C. Ralph and P. K. Lam, "Teleportation with Bright Squeezed Light," Phys. Rev. Lett. 81, 5668-5671 (1998).

[16] A. Zeilinger, "Experiment and the Foundations of Quantum Physics," Rev. Mod. Phys. 71, S288-S297 (1999).

[17] A. Peres, Quantum Theory: Concepts and Methods, (Kluwer, Dordrecht, 1993).

[18] C. A. Fuchs and C. M. Caves, "Mathematical Techniques for Quantum Communication Theory," Open Sys. and Info. Dyn. 3, 345-356 (1995).

[19] H. Barnum, C. M. Caves, C. A. Fuchs, R. Jozsa, and B. Schumacher, "Noncommuting Mixed States Cannot Be Broadcast," Phys. Rev. Lett. 76, 2818-2821 (1996).

[20] H. Reichenbach, Philosophic Foundations of Quantum Mechanics, (U. California Press, Berkeley, 1944), pp. 91-92.

[21] A. Vogt, "Position and Momentum Distributions Do Not Determine the Quantum Mechanical State," in Mathematical Foundations of Quantum Theory, edited by A. R. Marlow (Academic Press, New York, 1978), pp. 365-372.

[22] C. A. Fuchs, Distinguishability and Accessible Information in Quantum Theory, Ph. D. thesis, University of New Mexico, Albuquerque, NM (1996); see also quant-ph/9601020.

[23] C. A. Fuchs, "Just Two Nonorthogonal Quantum States," to appear in Quantum Communication, Computing, and Measurement 2, edited by P. Kumar, G. M. D'Ariano, and O. Hirota (Plenum Press, NY, 1998); see also quant-ph/9810032.

[24] H. Barnum, Quantum Information, Ph. D. thesis, University of New Mexico, Albuquerque, NM (1998). 
[25] L. Mandel and E. Wolf, Optical Coherence and Quantum Optics, (Cambridge U. Press, Cambridge, 1995).

[26] P. van Loock, S. L. Braunstein, and H. J. Kimble, "Broadband Teleportation," to appear in Phs. Rev. A; see also quant-ph/9902030.

[27] S. J. van Enk, "On Teleportation with Continuous Variables," to appear (1999).

[28] H. P. Yuen and M. Lax, "Multiple-Parameter Quantum Estimation and Measurement of Nonselfadjoint Observables," IEEE Trans. Inf. Theor. IT-19, 740-750 (1973).

[29] E. Arthurs and J. L. Kelly, Jr., "On the Simultaneous Measurement of a Pair of Conjugate Observables," Bell Syst. Tech. J. 44, 725-729 (1965).

[30] S. D. Personick, "An Image-Band Interpretation of Optical Heterodyne Noise," Bell Syst. Tech. J. 50, 213-216 (1971).

[31] H. M. Nussenzveig, Introduction to Quantum Optics, (Gordon and Breach, London, 1973).

[32] C. A. Fuchs, "Squeezing Quantum Information through a Classical Channel," in preparation. 